DOI 10.14409/fa.v21i1.11124

\title{
Obtención de híbridos de Portulaca umbraticola a través de cruzamientos convencionales
}

\author{
Sandí Montero, H. ${ }^{1} ;$ Zuñiga Orozco, A. ${ }^{2}$ \\ \& Carrodeguas González, A. ${ }^{3}$
}

\begin{abstract}
RESUMEN
Las especies del género Portulaca son muy utilizadas en jardinería para planta en maceta y para decoración de exteriores. Son fáciles de cultivar, poseen requerimientos mínimos, aportan belleza a un ambiente particular y constituyen un valorado recurso para el paisajismo. El mercado de plantas ornamentales es muy dinámico y se muestra ávido de incorporar frecuentemente nuevas variedades e híbridos. Por tales razones, el objetivo de la presente investigación es: crear nuevos híbridos de $P$. umbraticola a través de cruzamientos intraespecíficos. Se realizaron cruzamientos entre cuatro cultivares de P. umbraticola con flores de diferentes colores (blanca, roja, roja-fusia y bicolor amarillo-fusia) bajo un diseño de dialelo parcial sin incorporar autopolinizaciones. Después de realizadas las polinizaciones, se midió el porcentaje de formación de frutos, cantidad de semillas y porcentaje de germinación en cada cruzamiento. Se obtuvo en promedio un \% de formación de frutos de 42,5\%, 13,5 semillas por fruto y un \% de germinación de 22,5\%. Se obtuvieron híbridos con características morfológicas similares en cada cruzamiento, sin embargo, presentaron diferentes patrones de coloración interesantes en la flor y follaje. Todos los híbridos, excepto los del cuarto cruzamiento, mostraron cambios fenotípicos novedosos. También se analiza los posibles patrones de herencia para el color de flor. Al presentarse estos nuevos fenotipos pueden considerarse como material para desarrollar nuevos cultivares con interés ornamental y paisajístico. La hibridación intraespecífica de $P$. umbraticola es una técnica efectiva para el mejoramiento genético de la especie y la obtención de nuevos cultivares.
\end{abstract}

Palabras clave: hibridación, fitomejoramiento, morfología, ornamental, novedoso.

1.- 1.- Ingeniería Agronómica. UNED. CU Heredia, Costa Rica. Email: heyjo2bonn@yahoo.com https://orcid.org/0000-0003-1418-2119

2.- Ingeniería Agronómica. UNED. San Pedro, San José, Costa Rica. Email: azunigao@uned.ac.cr https://orcid.org/0000-0001-8214-4435

3.- Investigadora en mejoramiento genético vegetal. Email: ayerim2009@gmail.com

https://orcid.org/0000-0001-5890-4174

Manuscrito recibido el 10 de julio de 2021 y aceptado para su publicación el 16 de diciembre de 2021.

Sandí Montero H.; Zúñiga Orozco A. \& Carrodeguas González A. Obtención de híbridos de Portulaca umbraticola a través de cruzamientos convencionales

FAVE - Ciencias Agrarias 21 (1): 85-98 - CC BY-NC-SA 4.0 


\begin{abstract}
Obtaining hybrids of Portulaca umbraticola through conventional crossing. The species of the genus Portulaca are widely used in gardening for potted plants and for outdoor decoration. They are easy to plant, have minimal requirements, add beauty to a particular environment and are a valued resource for landscaping. The ornamental plant market is very dynamic and is eager to frequently incorporate new varieties and hybrids. For these reasons, the objective of this research is: to create new hybrids of Portulaca umbraticola through intraspecific crosses. Crossings were made between four cultivars of P. umbraticola with flowers of different colors (white, red, red-fusia and bicolor yellow-fusia) under a partial diallel design without incorporating self-pollinations. After the pollinations were carried out, the percentage of fruit set, the number of seeds and the percentage of germination were measured in each cross. An average of fruit formation percentage of $42.5 \%, 13.5$ seeds per fruit and a germination percentage of $22.5 \%$ were obtained. As a result, hybrids with similar morphological characteristics were obtained in each cross, however, they presented different interesting coloring patterns in the flower and foliage. All hybrids, except those of the fourth cross, showed novelty phenotypic changes. Possible inheritance patterns for flower color are also analyzed. By presenting these new phenotypes, they can be considered as material to develop new cultivars with ornamental and landscape interest. The intraspecific hybridization of P. umbraticola is an effective technique for the genetic improvement of the specie and obtaining new cultivars.
\end{abstract}

Key words: hybridization, plant breeding, morphology, ornamental, novel.

\section{INTRODUCCIÓN}

El género Portulaca pertenece a la familia Portulacacea y está compuesto por 200 especies de plantas anuales con origen en las regiones tropicales y subtropicales de América del Sur. Las especies más cultivadas como ornamentales son: P. grandiflora, P. umbraticola y P. pilosa (Maguvu et al., 2015). Las portulacas son muy utilizadas por su alto potencial ornamental y son ampliamente comercializadas como plantas florales anuales para jardín, especialmente P. umbraticola (Mera et al., 2011). También tienen un valor nutricional como alimento para el ser humano (Equilá, 2014).
Portulaca umbraticola se distribuye desde el sur de Estados Unidos e islas del Caribe hasta Sudamérica. Es una planta herbácea anual de raíces fibrosas. Presenta tallos postrados de $10-50 \mathrm{~cm}$ de largo, ramificados, carnosos, glabros, verdes o rojizos. Las hojas son alternas, aplanadas, con la lámina lanceolada, espatulada o estrechamente obovada. Exhibe inflorescencias terminales con una a seis flores de dos a cinco cm de diámetro, sustentadas por un involucro de cuatro a cinco hojas de 10-30 x 1-7 mm (Rodríguez, 2014).

Actualmente los mejoradores de plantas ornamentales emplean varios métodos que abarcan desde el mejoramiento tradicional 
hasta la transformación genética y técnicas de biología molecular (Gómez, 2020).

La hibridación es un método comúnmente utilizado para obtener variabilidad genética en todo tipo de plantas, incluyendo las del género Portulaca (Ponce et al., 2014). En los programas de mejoramiento genético de plantas de interés ornamental es importante contar con una amplia base genética que garantice gran cantidad de variabilidad para aumentar las probabilidades de seleccionar nuevos genotipos (Rubio y López, 2015). Messmer et al. (2015) mencionan que este tipo de programas consisten de tres fases: generación de la variabilidad genética, selección de genotipos y evaluación de los genotipos seleccionados con caracteres agronómicos de interés.

La hibridación interespecífica se pone en práctica cuando se cruzan plantas de diferentes especies. Este método brinda un enorme potencial para aprovechar la herencia transgresiva en los caracteres cuantitativos y cualitativos, además permite la obtención de genotipos con mayores ventajas en cuanto a adaptación a diferentes ambientes (Camarena et al., 2014).

A pesar de que la hibridación es un método ampliamente aplicado, la obtención de híbridos interespecíficos se encuentra en muchas ocasiones limitada por diferentes barreras de incompatibilidad, como anomalías en la polinización, interrupción del crecimiento del tubo polínico, barreras poscigóticas, desarrollo anormal del endospermo, variación en el número de cromosoma de especies parentales y desarrollo anormal de semillas (Milicia et al., 2018). Sin embargo, en la hibridación intraespecífica, las barreras de incompatibilidad son menos rígidas, por lo que muchas veces se recurre a esta para generar variabilidad y obtener vigor híbrido. Este tipo de hibridación ocu- rre cuando se cruzan plantas de diferentes cultivares o distintos genotipos que pertenecen a una misma especie (Ghani et al., 2020, Víquez, 2010).

Debido a la importancia de obtener nuevos genotipos y variedades de $P$. umbraticola para el mercado ornamental, el objetivo de este estudio es crear nuevos híbridos de $P$. umbraticola a través de cruzamientos intraespecíficos.

\section{METODOLOGÍA}

\section{Ubicación y duración del estudio}

El estudio se realizó en la localidad de Santo Tomás de Santo Domingo de Heredia, Costa Rica, coordenadas 958’42.3”N, $84^{\circ} 4^{\prime} 33.81^{\prime \prime} W$, entre los meses de julio del 2020 y mayo del 2021.

El clima de Santo Tomás de Santo Domingo de Heredia es templado, tiene una temperatura promedio de $23^{\circ} \mathrm{C}$, una altura 1242 m.s.n.m, con un promedio de precipitaciones de $3084 \mathrm{~mm}$ anuales.

\section{Material vegetal parental}

Se utilizaron como parentales, cultivares de P. umbraticola con flores de color blanco, naranja, rojo, fucsia y amarillo con fucsia (bicolor), actualmente disponibles en el mercado local. Se emplearon 30 clones, reproducidos por esquejes de todos los cultivares antes mencionadas con el fin de contar con varias plantas y aumentar la probabilidad de contar con flores abiertas para las polinizaciones.

\section{Cruzamientos}

Para este estudio se estableció un dialelo parcial mediante la realización de bloques de cruzamiento entre los cultivares de $P$. umbraticola. Se realizaron 10 polinizacio- 
nes por cruzamiento. En la figura 1 se muestran los parentales utilizados en cada cruce.

Los parentales se plantaron en macetas de plástico de 1,4 L con un sustrato constituido por tierra negra con granza y se abonó con lombricompost. Se establecieron los bloques de cruzamiento según los caracteres presentes que se deseaban obtener en la progenie. Las polinizaciones se realizaron de forma manual con el uso de una lupa y una aguja, con la cual se recogió el polen de la flor donadora y se aplicó en el pistilo de la flor receptora. Luego de cada polinización se desinfectó la aguja con alcohol. Las plantas se distribuyeron en el invernadero ordenadamente de acuerdo a los cruces que se realizaron.
Manejo de la producción de frutos y semillas de P. umbraticola

Luego de la polinización manual de cada flor de $P$. umbraticola, se marcó una parte de la base de la flor con pintura acrílica, porque cada tallo produce varias flores y no todas abren al mismo tiempo. Posteriormente, se cubrieron las flores con bolsas de nylon para proteger el futuro fruto y evitar que algún insecto pudiera dañar la polinización. Se registró el número de frutos obtenidos por tipo de cruzamiento y el número de semillas por fruto.

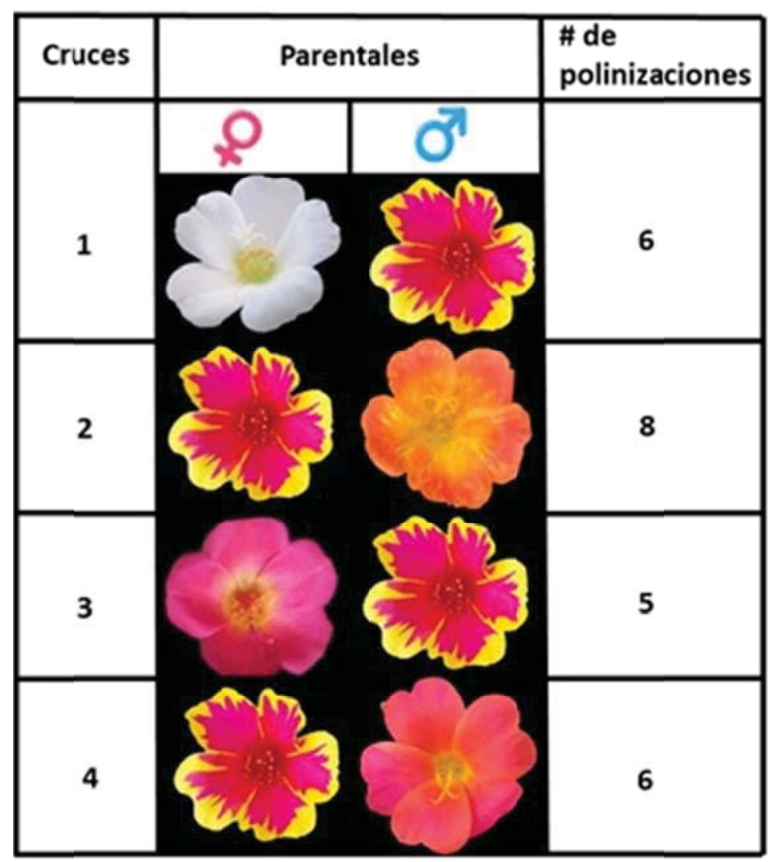

Figura 1. Cultivares de Portulaca umbraticola utilizados como parentales en los cuatro cruces realizados.

Figure 1. Cultivars of Portulaca umbraticola used as parents in the four crosses made. 


\section{Cuantificación de germinación por cruce}

Una vez que fueron recolectadas todas las semillas de todos los cruces, se pusieron a germinar en bandejas de plástico transparente con tapa con sustrato de turba, el cual se humedeció con agua utilizando un pulverizador. Y se colocaron en un lugar luminoso, pero sin la incidencia del sol directo y se controló la humedad dentro de la bandeja. Se calculó el porcentaje de germinación según cada cruce: \% germinación = (plántulas germinadas/semillas plantadas) *100. Luego de germinadas las semillas, se retiró la tapa de la bandeja y se colocó gradualmente bajo el sol directo.

\section{Manejo de progenies}

Cuando las plántulas alcanzaron unos 3 cm de altura y presentaron seis hojas (alrededor del mes y medio después de la siembra de la semilla) se plantaron en las macetas con volumen de 0,9 L. Un mes después se trasplantaron a las macetas con volumen de $1,8 \mathrm{~L}$, con sustrato constituido con tierra negra y granza en proporción 1:1. Se utilizó una fuente de fertilizantes N-P-K (3010-10) granulada de la marca Evergreen(R) (fabricado en Costa Rica) aplicado a la siembra para acelerar el crecimiento. Luego se aplicó una fuente con alto contenido en fósforo N-P-K (10-50-10) granulado (5 $\mathrm{g} / \mathrm{L}$ ), de la misma marca para estimular la floración. Cada 15 días se aplicaron dos tipos de insecticidas alternados según presencia de insectos con los siguientes ingredientes activos: cipermetrina EC (1 L/ha) y benfuracarb EC (1 L/ha).

\section{Descripción de los híbridos}

A los 45 días de siembra, los híbridos obtenidos se describieron mediante variables cualitativas y cuantitativas al momento de la floración. Entre las variables cualitativas, se tomaron en cuenta el porte, tipo de hoja, tipo de borde de hoja, forma de base de hoja, disposición de la hoja, color de hoja, color de las flores, presencia de ojo en el centro de la flor, color de estigma y distribución de color de la flor. Entre las cuantitativas el ancho de pétalo, diámetro de cápsula, diámetro de la planta, longitud de hoja, diámetro de flor y longitud del pétalo. Se midieron en total 10 flores de cada uno de los híbridos, para estas medidas se utilizó una regla en cm y además los colores se determinaron con la carta de color de la Royal Horticultural Society Colour Chart (Royal Horticulture Society, 2018).

\section{RESULTADOS}

En la tabla 1 se muestran los datos relacionados a la polinización y producción de semillas en los cuatro cruces intraespecíficos de P. umbraticola. En las variables cuantificadas se obtuvo en promedio los siguientes valores: formación de frutos 42,5\% (Min 30\%, Max 60\%), 13,5 semillas por fruto y un \% de germinación de 22,5\% (Min 15\%, Max 25\%).

En la tabla 2 se muestra la descripción detallada de las variables morfológicas cuantificadas en los híbridos obtenidos. 
H. Sandí Montero et al.

Tabla 1. Porcentaje de formación de frutos, producción de semillas y germinación en cuatro cruces intraespecíficos de P. umbraticola.

Table 1. Percentage of fruit set, seed production and germination in four intraspecific crosses of P. umbraticola.

\begin{tabular}{|c|c|c|c|c|}
\hline Cruce & $\begin{array}{c}\text { \% formación } \\
\text { de frutos }\end{array}$ & $\begin{array}{c}\mathrm{N}^{\circ} \text { de semillas } \\
\text { obtenidas por } \\
\text { fruto }\end{array}$ & $\begin{array}{c}\mathrm{N}^{\circ} \text { de } \\
\text { plántulas } \\
\text { obtenidas }\end{array}$ & $\begin{array}{c}\% \text { de } \\
\text { germinación }\end{array}$ \\
\hline 1 & 60 & $15 \pm 2$ & 3 & 20 \\
\hline 2 & 40 & $13 \pm 1$ & 2 & 15 \\
\hline 3 & 30 & $12 \pm 1$ & 3 & 25 \\
\hline 4 & 40 & $14 \pm 2$ & 4 & 30 \\
\hline
\end{tabular}

Tabla 2. Descripción morfológica de los híbridos obtenidos en cuanto a caracteres de interés ornamental en P. umbraticola. (versión en inlgés: Morphological description of the hybrids obtained regarding to characters of ornamental interest in P. umbraticola).

Table 2. Morphological description of the hybrids obtained in traits of ornamental interest in $P$. umbraticola.

\begin{tabular}{|c|c|c|c|c|}
\hline \multirow[b]{2}{*}{ Caracter } & \multicolumn{4}{|c|}{ FIDTाao } \\
\hline & Starblush & Tekila Sunset & Coral Lipstick & Golden Mirror \\
\hline Porte & Semierecto & Semierecto & Semierecto & Rastrero \\
\hline Diámetro Planta $(\mathrm{cm})$ & $34 \pm 2,3$ & $35 \pm 1,8$ & $34 \pm 3,1$ & $33 \pm 2,5$ \\
\hline Tipo Hoja & obovadas & obovadas & obovadas & obovadas \\
\hline Tipo de Borde Hoja & Entero & Entero & Entero & Entero \\
\hline $\begin{array}{l}\text { Forma de Base de } \\
\text { Hoja }\end{array}$ & Agudo & Agudo & Agudo & Agudo \\
\hline Long. Hoja (cm) & $2,5 \pm 0,3$ & $2 \pm 0,2$ & $1,5 \pm 0,3$ & $2,5 \pm 0,4$ \\
\hline Disposición de Hoja & Alterna & Alterna & Alterna & Alterna \\
\hline Color Hoja (RHS) & $\begin{array}{c}\text { 140B con bordes } \\
53 \mathrm{~A} \text {. Algunas } \\
\text { hojas pueden } \\
\text { tener } 53 \mathrm{~A} \text { en el } \\
50 \% \text { del área }\end{array}$ & $\begin{array}{c}140 \mathrm{~B} \text { con bordes } \\
53 \mathrm{~A}\end{array}$ & $\begin{array}{c}140 \mathrm{~B} \text { con bordes } \\
53 \mathrm{~A} \text { y variegadas } \\
\text { con blanco }\end{array}$ & $140 B$ \\
\hline $\begin{array}{l}\text { Presencia de ojo en } \\
\text { centro de la flor }\end{array}$ & Ausente & Ausente & Presente & Ausente \\
\hline Diámetro de flor $(\mathrm{cm})$ & $3,1 \pm 0,2$ & $3,5 \pm 0,2$ & $2,2 \pm 0,3$ & $2,5 \pm 0,3$ \\
\hline Long. Pétalo (cm) & $1,5 \pm 0,1$ & $1,5 \pm 0,2$ & $1 \pm 0,2$ & $1 \pm 0,1$ \\
\hline Color estigma (RHS) & $\begin{array}{l}\text { 75D (purple-violet- } \\
\text { blue) }\end{array}$ & $\begin{array}{l}\text { N82A (purple- } \\
\text { violet-blue) }\end{array}$ & $\begin{array}{l}\text { N82A (purple- } \\
\text { violet-blue) }\end{array}$ & $\begin{array}{l}\text { 5B (yellow- } \\
\text { orange-red) }\end{array}$ \\
\hline $\begin{array}{l}\text { Distribución de color } \\
\text { en flor }\end{array}$ & No uniforme & No uniforme & Uniforme & Uniforme \\
\hline Color flor (RHS) & $\begin{array}{l}\text { 57B (purple-violet- } \\
\text { blue) con patrones } \\
\text { diversos } \\
\text { matizados con } \\
\text { fondo blanco y } \\
\text { líneas } \\
\text { longitudinales de } \\
57 \mathrm{~B}\end{array}$ & $\begin{array}{l}\text { 5B y borde N25B } \\
\text { (yellow-orange- } \\
\text { red) }\end{array}$ & $\begin{array}{l}\text { 57B (purple- } \\
\text { violet-blue) }\end{array}$ & $\begin{array}{l}\text { 5B (yellow- } \\
\text { orange-red) }\end{array}$ \\
\hline
\end{tabular}


De los cuatro cruces realizados entre dis- la progenie mostró características similares tintos cultivares de $P$. umbraticola, se obtuvieron tres híbridos para el cruce 1 , dos para el cruce 2, tres para el cruce 3 y 4 para por lo cual en la Figura 2 se observa la flor y hoja de un ejemplar de cada cruce, adeel cuarto cruce. Dentro de cada cruce toda más en la tabla 2 se hace una descripción detallada de los mismos.

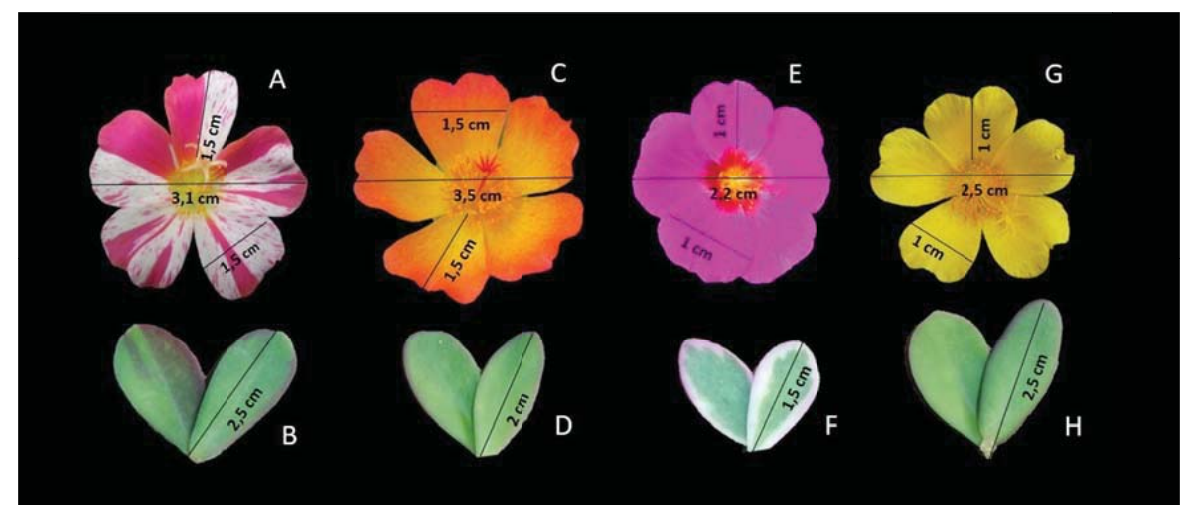

Figura 2. Híbridos obtenidos en los cuatro cruzamientos realizados en P. umbraticola A y B: Flor y hojas del híbrido "Starblush" obtenido en el cruce 1: cuyos parentales son la portulaca de flor blanca con la portulaca de flor combinada amarillo/fucsia; C y D: Flor y hojas del híbrido "Tekila Sunset" producto del cruce 2: cuyos parentales son la portulaca de flor combinada amarillo/fucsia con la portulaca de flor anaranjada; E y F: Flor y hojas del híbrido "Coral Lipstick" obtenido en el cruce 3: cuyos parentales son la portulaca de flor roja/fucsia con la portulaca de flor combinada amarillo/fucsia; G y H: Flor y hojas del híbrido "Golden Mirror" obtenido en el cruce 4: cuyos parentales son la Portulaca de flor combinada amarillo/fusia con la portulaca de flor roja. Los nombres de los híbridos son propuestos y están en proceso de inscripción.

Figure 2. Hybrids obtained in the four crosses made in portulaca umbraticola A and B: Flower and leaves of the hybrid "Starblush" obtained in crossing 1: whose parents are the portulaca with a white flower with the portulaca with a combined yellow / fuchsia flower; C and D: Flower and leaves of the hybrid "Tekila Sunset" product of crossing 2: whose parents are the Portulaca with a combined yellow / fuchsia flower and the portulaca with an orange flower; E and F: Flower and leaves of the hybrid "Coral Lipstick" obtained in crossing 3: whose parents are the portulaca with a red / fuchsia flower and the portulaca with a combined yellow / fuchsia flower; G and H: Flower and leaves of the hybrid "Golden Mirror" obtained in crossing 4: whose parents are the Portulaca with a combined yellow / fusia flower and the portulaca with a red flower. The names of the hybrids are proposed and are in registration process. 

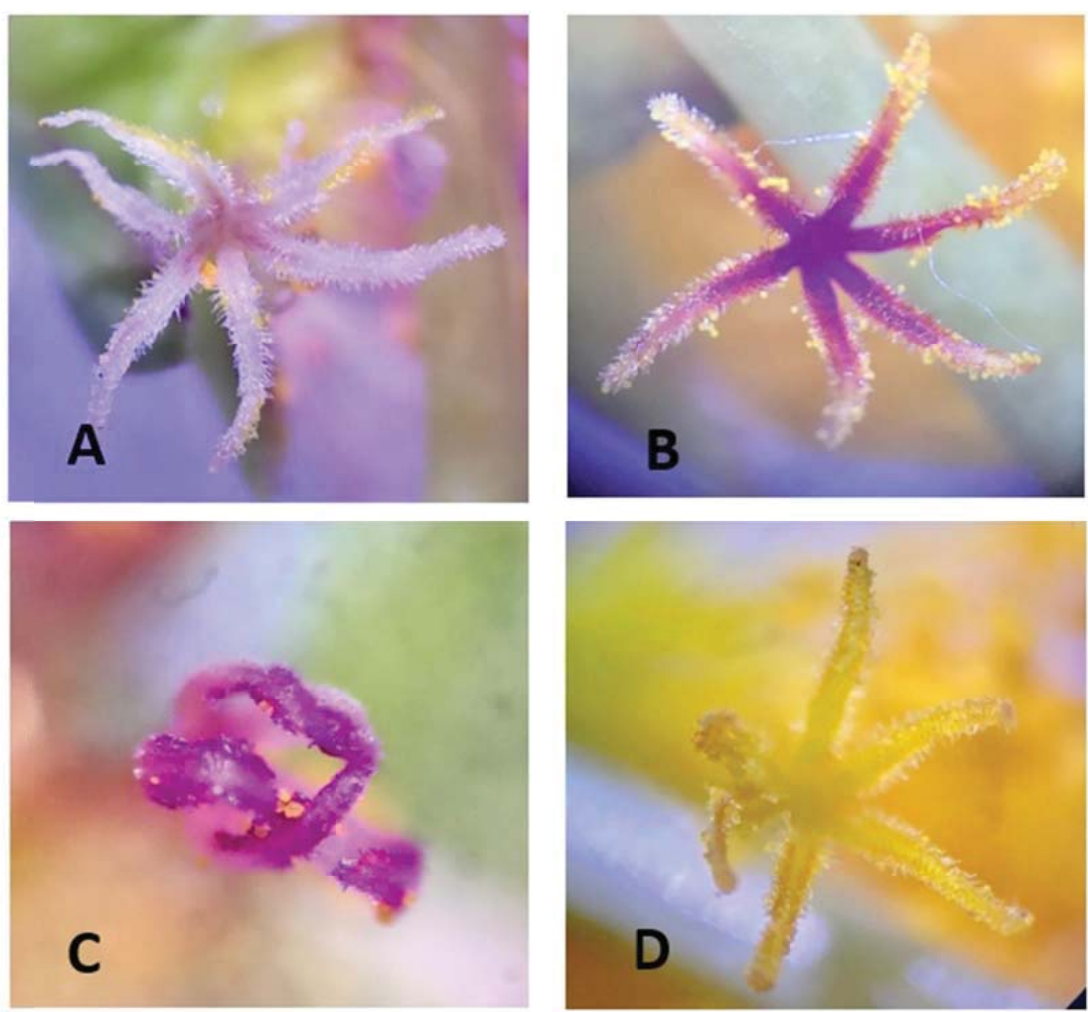

Figura 3. Estilo del estigma de las flores de los cuatro híbridos de P. umbraticola. A: híbrido Starblush obtenido en el cruce 1. B: híbrido Tekila Sunset producto del cruce 2. C: híbrido Coral Lipstick obtenido en el cruce 3. D: Flor y hojas del híbrido Golden Mirror obtenido en el cruce 4. Figure 3. Flower stigma style of the four hybrids of P. umbraticola. A: Starblush hybrid obtained in crossing 1. B: Tekila Sunset hybrid product of crossing 2. C: Coral Lipstick hybrid obtained in crossing 3. D: Flower and leaves of the Golden Mirror hybrid obtained in crossing 4. 
En el híbrido obtenido en el cruce 1 (Starblush) presentado en la figura 2A y 2B se observó un cambio significativo en el color de la flor con respecto a sus parentales. En este híbrido permaneció el color blanco de la hembra y del macho heredó vetas de color fucsia. En cuanto a las hojas se heredó la pigmentación verde de la madre con pequeñas manchas en un tono verde más claro. Presenta una emarginación poco profunda entre los pétalos. El estilo se presentó dividido en 6 estigmas lineales ciliados de color rosa claro (Figura 3A).

Para los híbridos del cruce 1 (Starblush) se presentaron flores con patrones de coloración variable incluso dentro de la misma planta como se aprecia en la Figura 4.

En la flor del híbrido obtenido en el cruce 2 (Tekila Sunset), presentado en la figura 2C y 2D se heredaron levemente los dos colores de la hembra (amarillo y fucsia), así como el color anaranjado del macho, aunque en el híbrido levemente más claro. El centro de la flor del híbrido presentó un color amarillo y a medida que se van observando los bordes, estos se vuelven de color naranja oscuro. Las hojas presentaron el mismo tono de verde que los parentales, sin embargo, los bordes tuvieron tonalidades color vino oscuro. En el híbrido, la flor fue ligeramente más grande que los parentales, 0,5 cm aproximadamente. La flor presentó una emarginación medianamente profunda. Las hojas de este híbrido presentaron coloración verde con el borde color rojizo. Las cápsulas midieron 3,4 mm de largo. El estilo estuvo dividido en 6 estigmas lineales ciliados de color violeta en la base y con tonalidades rosa hacia los extremos (Figura 3B).

En la Figura 2E y 2F se muestra el híbrido producto del cruce 3 (Coral Lipstick), se heredó el color fucsia de la flor de la hembra y las hojas presentan variegación. La flor presentó un borde interno color rojizo, lo cual no se había observado en sus parentales. Una característica muy distintiva de este híbrido en comparación a los otros obtenidos fue que las hojas se presentaron color verde, pero con el borde externo blanco y una coloración fina color fucsia. Las cápsulas midieron 3,1 mm de largo. El estilo se dividió en 6 estigmas lineales ciliados de color rosa oscuro, pero a diferencia de los otros híbridos, estos permanecen cerrados (Figura 3C).

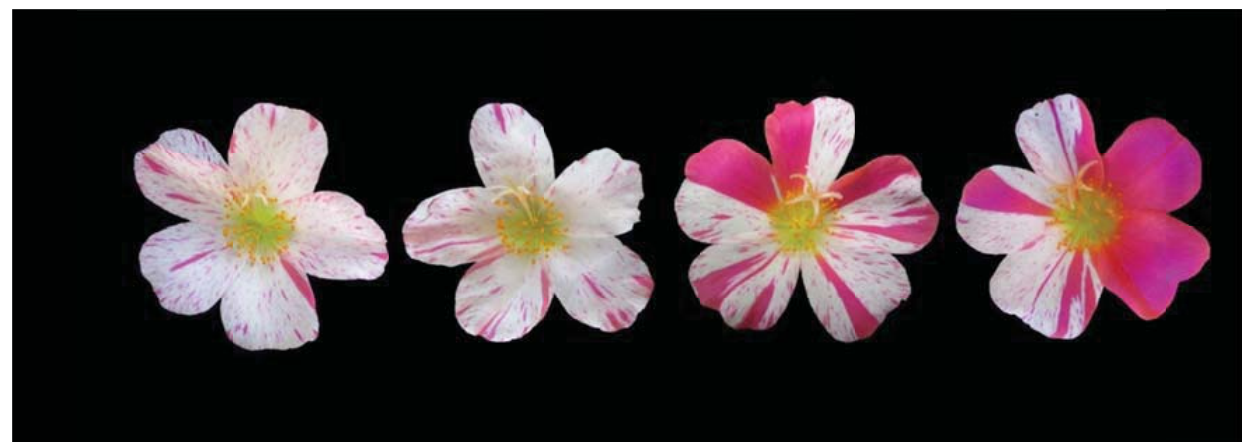

Figura 4. Diferentes patrones de coloración de las flores de los híbridos del cruce 1 (Starblush). Figure 4. Different color patterns of the flowers of the cross 1 hybrids (Starblush). 
En la figura 2G y 2H se observa el híbrido del cruce 4 (Golden Mirror), presentó una flor simple con solapamiento medio de los pétalos. Las cuatro plantas que germinaron conservaron el color amarillo único en sus flores uniformemente. Las hojas presentaron una coloración verde con el borde externo color marrón rojizo. Las cápsulas midieron 3,2 mm de largo. El estilo se dividió en 6 estigmas lineales ciliados de color amarillo (Figura 3D).

\section{DISCUSIÓN}

Mediante el cruzamiento se obtuvieron híbridos que presentaron algunas características no mostradas en sus parentales.

Portulaca umbraticola se reporta como una especie alógama con autogamia parcial (Aizen 1993, Aizen y Feinsinger, 1994). Las especies alógamas como la mayoría en el género Portulaca, se caracterizan por un alto porcentaje de formación de frutos debido a la polinización cruzada. La frecuencia de hibridación es mayor en las especies alógamas como en P. umbraticola que en autógamas, estas últimas presentan poblaciones formadas por una sola línea pura o una mezcla de líneas puras (Angulo y Ortiz, 2020).

En la presente investigación el porcentaje de frutos obtenidos y el número de semillas por cápsula es menor que el reportado en la literatura para esta especie en cruces intraespecíficos. Wickramasinghe et al. (2009) obtuvieron un promedio de 28,1 semillas por cápsula y un éxito de formación de frutos del $75 \%$ respectivamente, mientras en esta investigación se obtuvo un promedio de 13,5 semillas y $42,5 \%$ de forma- ción de frutos. Lo anterior puede deberse a las diferentes condiciones experimentales, un tamaño de muestra pequeño y al material utilizado.

Las condiciones experimentales pueden variar los resultados en tanto que las diferencias pueden ser significativas, sobre todo cuando se utilizan diferentes genotipos de diversos orígenes y distancias genéticas. Por su parte también el efecto heterótico tiene gran influencia en los resultados. En cultivos ornamentales no hay tanta investigación publicada al respecto, sin embargo, en cultivos hortícolas como tomate hay en la literatura muchos reportes. De acuerdo al contexto anterior, Burbano y Vallejo (2017) reportan, para la variable peso de frutos por planta un rango de 2,7-4,6 kg, mientras Martínez-Vásquez et al. (2016) reportan 0.9-3.6 kg. Lo anterior se debe a los parentales utilizados en los cruzamientos y un rango de heterosis reportado entre -21.8 y $111.2 \%$, sin embargo, los autores también reportan condiciones experimentales distintas en cuanto a localidad, temperatura, humedad relativa, precipitación anual, tipo de trasplante, sustrato y distancia de siembra.

Otro de los factores que pudo haber influido en las diferencias en la obtención de frutos y semillas con respecto a otros estudios fue el método de cruzamiento. En este estudio se realizó solamente en un sentido y no fue recíproco, sin embargo, esto se realizó intencionalmente debido a que se quería conocer los resultados en un único sentido. En otros cultivos ornamentales del género Mercadonia se han realizado cruces recíprocos y se han obtenido diferencias debido al genotipo de los parentales y también a una alogamia parcial. Lo anterior pone de manifiesto que el método de cruzamiento 
y el tipo de sistema reproductivo tienen influencia en los resultados de las variables estimadas (Greppi, 2017) y es de esperar que en el presente estudio este factor esté integrado dentro de las estimaciones.

Los nuevos cultivares de $P$. umbraticola generalmente son bien aceptados en el mercado de plantas ornamentales y usualmente se propagan vía asexual con el objetivo de mantener las características deseadas. El mercado ornamental también exige la comercialización de semillas, pero para asegurar que se mantengan las características de los parentales, es necesario contar con líneas puras. La manera convencional de crear líneas homogéneas es mediante la autopolinización, la cual es posible en esta especie, pero trae algunas desventajas como la inducción de la senescencia temprana en la flor (Aizen, 1993). Además, mediante autopolinización se produce baja cantidad de semilla y bajo porcentaje de germinación. Por lo explicado anteriormente, se debería aumentar la cantidad de polinizaciones para obtener mayor cantidad de semilla y seleccionar solamente progenies con el mayor porcentaje de germinación.

En poblaciones naturales, la hibridación posee una importancia ecológica y evolutiva como mecanismo de especiación y diversificación en las plantas (Ramírez y Chávez, 2014). En el caso del mejoramiento genético, la hibridación permite el desarrollo de poblaciones segregantes para la posterior selección de nuevos genotipos. En el presente estudio, los híbridos obtenidos son genotipos promisorios para el posterior desarrollo de variedades debido a que presentan un alto potencial ornamental por su aspecto llamativo en el color de las flores y el follaje. Camarena et al. (2014) señalan que para mejorar el valor estético de las plantas se toman en cuenta diversos criterios tales como, variegaciones, nuevos portes, follaje con patrones de coloración variable, flores de colores novedosos, una mayor productividad en cuanto a cantidad de flores y longevidad de las mismas.

Existen pocos estudios científicos sobre la hibridación en el género Portulaca. Wickramasinghe et al. (2009) realizaron hibridación con varias especies (P. grandiflora, P. umbraticola, Calandrinia balonensis) y determinaron que es posible lograr híbridos intraespecíficos, lo cual concuerda con los resultados obtenidos en esta investigación. Los autores mencionan también que no fue posible obtener híbridos intergenéricos debido a barreras de prefertilización. Además, se encontró que la emasculación en cruces intraespecíficos produce un efecto negativo en esta especie, por lo cual es un factor a considerar.

En cuanto a la herencia de caracteres, es difícil determinarlos con certeza debido al bajo porcentaje de germinación, lo cual provoca poca cantidad de plantas y por ende es difícil observar si hubo alguna segregación. Por otra parte, un posible patrón de coloración en el híbrido 1 (Starblush) se presentó con una posible codominancia entre el color blanco y el fucsia. La codominancia ocurre cuando ambos alelos producen efectos distinguibles en el fenotipo (NCBI, 2021). En la opinión de los autores, para el caso de los diferentes patrones de coloración, también existe un efecto ambiental, puesto que, plantas del mismo genotipo presentan mayor coloración blanca cuando son sembradas a la sombra y mayor pigmentación fucsia cuando se exponen al sol directo. Lo anterior se explica porque los pigmentos como las antocianinas se ven estimulados a expresarse para proteger a las flores de los rayos del sol (Lodish et al., 2016). 
En el híbrido 2 (Tekila Sunset) se observó una diferenciación entre la coloración de la parte interna y el borde de la flor, lo cual podría indicar una posible segregación independiente de genes por zonas. En las flores, diferentes niveles y composiciones de antocianinas son responsables de la variación de color en el cuerpo del pétalo, incluidos los pigmentos rojos y rosados de cianidina y peonidina; pigmentos rosados de pelargonidina; y pigmentos rosados oscuros, lavanda y azul de delfinidina, petunidina y malvidina (Kim et al., 1989). Por lo anterior es deducible que, los genes relacionados con la vía de la síntesis de los diferentes pigmentos en los pétalos segregan independientemente. Este comportamiento ha sido reportado en otras plantas como Hibiscus radiatus (Lattier y Contreras, 2020).

Para el caso del híbrido 3 (Coral Lipstick) se observó una aparente dominancia completa del color fucsia sobre el bicolor fucsia/amarillo, sin embargo, en este caso se presentó una variegación en la totalidad del follaje (verde/blanco/fucsia). La variegación ocurre en distintas partes de las hojas, la más común es la que se manifiesta en los bordes y de esta forma se desarrollan hojas verdes con ausencia de pigmentación en los márgenes (Paredes, 2020). La variegación blanca ocurre por la falta de capacidad de la planta para producir cualquier tipo de pigmento en esa área y los matices de color rosado, rojo o púrpura se deben al resultado del pigmento antocianina, estos factores hacen que las plantas variegadas tengan un alto valor ornamental, gran potencial de uso y comercialización (Duarte, 2018).

La variegación puede tener un origen genético o ambiental, en este último caso se puede perder fácilmente y que las plantas recuperen la coloración verde por comple- to (Yu et al., 2007). Si la variegación es de origen ambiental sucede que al propagar un cultivar por semilla, se pierde esta característica, lo que obliga en este caso para mantener la variegación, realizar propagación asexual (Camarena et al., 2014). La variegación de origen genético es una característica descrita como recesiva y en el caso de monocotiledóneas como trigo y arroz se ha estudiado el mecanismo molecular implicado, se conoce que se produce por un defecto en el desarrollo de los cloroplastos (Xing et al., 2010).

En el híbrido producto del cruce 4 (Golden Mirror), no se obtuvo características novedosas. Sin embargo, la flor es ligeramente más grande y compacta que el cultivar de Portulaca amarilla común en los jardines.

\section{CONCLUSIONES}

Se obtuvieron fenotipos novedosos no registrados en el mercado ornamental para esta especie. Los híbridos intraespecíficos obtenidos se pueden propagar asexualmente para su comercialización.

En la presente investigación el porcentaje de formación de frutos obtenido, el número de semillas por cápsula y porcentaje de germinación fue menor que el reportado en la literatura para esta especie en cruces intraespecíficos.

Se recomienda estabilizar líneas hacia homocigosis para explorar la posibilidad de comercializar por semilla. 


\section{BIBLIOGRAFÍA}

Aizen M. 1993. Self - pollination shortens flower lifespan in Portulaca umbraticola H.B.K (Portulacaceae). Int. J. Plant Sci .154: 412-415.

Aizen MA, Feinsinger P. 1994 Forest fragmentation, pollination, and plant reproduction in a Chaco dry forest, Argentina. Ecology. 75: 330-351. doi:10.2307/1939538.

Angulo I, Ortiz MA. 2020. Mejoramiento genético en plantas alógamas y autógamas. Colombia: Universidad Nacional de Colombia. $23 \mathrm{pp}$.

Burbano E, Vallejo C. 2017. Producción de líneas de tomate "chonto", Solanum lycopersicum Mill., con expresión del gen sp responsable del crecimiento determinado. Revista Colombiana de Ciencias Horticolas. 11(1): 63-71.

Camarena F, Chura J, Blas, RU. 2014. Mejoramiento genético y biotecnológico de plantas. Perú: UNALM. 286 pp.

Duarte FP. 2018. Diferencias fisiológicas y cambios en los patrones de metilación del ADN durante la micropropagación de plantas albinas de Agave angustifolia Haw. [Tesis de doctorado]: Centro de Investigación Científica de Yucatán, México. https:// cicy.repositorioinstitucional.mx/jspui/bitstream/1003/1371/1/PCB_D_2018_Tesis_Fatima_Duarte.pdf.

Maguvu TE, Shimizu-Yumoto H, Shibata M. 2015. Difference in Flower Longevity and Endogenous Ethylene Production of Portulaca umbraticola Cultivars. The Hort. Jrnl. https://doi.org/10.2503/hortj.MI-086.

Equilá G. 2014. Cultivo y aprovechamiento de la verdolaga como planta nutritiva. [Tesis de Licenciatura]: Universidad de San Carlos, Guatemala. http://biblioteca.usac.edu.gt/ EPS/07/07_5249.pdf.
Ghani MA, Abbas MM., Ziaf KM, Azam M, Ali B, Amjad M, Anjum R, Noor A, Zahid M. 2020. Production and characterization of inter and intraspecific hybridization eggplant. Hortic. Bras. 38 (4): 407-414.

Gómez C. 2020. La edición de genomas para la mejora vegetal. Métode. 104 (45): 45-49.

Greppi J. 2017. Estudio de compatibilidad reproductiva en especies de Mecardonia nativas de Argentina. [Tesis de Maestría]: Universidad Nacional de Lomas de Zamora, Buenos Aires, Argentina. http://agrarias.unlz.edu.ar/ web18/wp-content/uploads/2020/06/Trabajo-Final_Juli\%C3\%A1n-Greppi.pdf.

Kim JH, Okubo H, Fujieda K, Uemoto S. 1989. Anthocyanin pigmentation patterning in petals of Hibiscus syriacus. J. Fac. Agr.Kyushu Univ. 33:253-258.

Lattier J., Contreras R. 2020. Segregation of Flower Color and Eyespot in Althea. Journal of the American Society for Horticultural Science. 145. 1-13. 10.21273/JASHS04824-19.

Lodish H, Berk A, Kaiser C, Krieger M, Bretscher A, Ploegh H, Matsudaira P, Darnell J. 2016. Cap 12. Cellular energetics. In: Molecular Cell Biology. Ed. W.H Freeman and Company. NY, USA.

Martínez-Vázquez E, Lobato-Ortiz R, García-Zavala J, Reyes-López D. 2016. Heterosis de cruzas entre lineas de tomate (Solanum lycopersicum L.) nativo mexicano tipo pimiento y lineas tipo saladette. Revista Fitotecnia Mexicana. 39(1): 67-77.

Mera LM, Bye RA, Castro D, Villanueva C. 2011. Documento de diagnóstico de Portulaca oleracea L. México: Universidad Autónoma Chapingo. 49 pp.

Messmer M, Wilbois K, Baier C, Scháfer F, Arncken C, Drexter D, Hildermann I. 2015. Técnicas de mejora vegetal. España: SEAE. $48 \mathrm{pp}$. 
Milicia VJ, Chiesa A, Coviella A, Soto, MS. Víquez A. 2010. Compatibilidad sexual en 2018. Producción de híbridos interespecíficos entre Nierembergia ericoides y Nierembergia linariaefolia mediante la aplicación de técnicas de rescate embrionario. Chil. J. Agric. Anim. Sci. 34(3): 234-242.

NCBI (2021). Glossary. National Human Genome Research Institute. USA Gov. https:// www.genome.gov/es/genetics-glossary/Codominancia.

Paredes W. 2020. Mejoramiento genético en plantas. Arequipe, Perú. Ed. Universidad Nacional San Agustín. 120 pp.

Ponce O, Rodríguez A, Hernández E, Leiva AT, Pérez L. 2014. Manual de técnicas de cultivo de plantas ornamentales. Cuba: Jardín Botánico Nacional. 132 pp. https://docplayer. es/114201372-Manual-de-tecnicas-de-cultivo-de-plantas-ornamentales.html

Ramírez G, Chávez JL. 2014. Mejoramiento genético de ornamentales del Estado de México. México: ICAMEX. 19 pp. https://icamex. edomex.gob.mx/sites/icamex.edomex.gob. mx/files/files/publicaciones/2014/ORNAMENTALES.pdf.

Rodríguez C. 2014. Estudio comparativo de la propagación y el efecto de la radiación lumínica, en una variedad comercial y una población natural de Portulaca oleracea L. [Tesis de Licenciatura]: Universidad de Sevilla, España. https://hdl.handle.net/11441/72586.

Royal Horticulturae Society. 1995. RHS colour chart. Royal Horticulture Society, Londres.

Rubio JR, López MJ. 2015. Agrojardinería y composiciones florales. Volumen I Agrojardinería y cultivo de plantas ornamentales. España: Editorial Síntesis S. A. 207 pp. cruces intraespecíficos e interespecíficos de Penstemon sp. [Tesis de Licenciatura]: Instituto Tecnológico de Costa Rica, Costa Rica. https://hdl.handle.net/2238/650.

Wickramasinghe P, Harrison D, Johnson M. 2009. Reproductive biology and intergeneric breeding compatibility of ornamental Portulaca and Calandrinia (Portulacaceae). Australian Journal of Botany. 57: 697-707.

Xing S, Miao J, Li S, Qin G, Tang S, Li H, et al. 2010. Disruption of the 1-deoxy-D-Xylulose-5-phosphate reductoisomerasa (DXR) gene results in albino dwarf and defects in trichome initiation and stomata closure in Arabidopsis. Cell Research, 20: 688-700.

Yu F, Fu A. Aluru M. Park S, Xu Y, Liu H, et al. 2007. Variegation mutants and mechanisms of chloroplast biogenesis. Plant, Cell \& Environment, 30: 350-365. https://doi.org/10.1111/j.1365-3040.2006.01630.x 